\title{
Sliding Model Control with Upper Bound Adaptive Learning for Virtual Axis Machine Tool
}

\author{
Yu Zhao ${ }^{1}$, Yongfeng $\mathrm{Cui}^{2,{ }^{*}}$ and Miaochao $\mathrm{Chen}^{3}$ \\ ${ }^{1}$ School of Basic Education, Jiangsu Food \& Pharmaceutical Science College, \\ Huaian, 223000, P. R. China \\ ${ }^{2}$ College of Network Engineering, Zhoukou Normal University, Zhoukou 466001, \\ P. R. China \\ ${ }^{3}$ School of Applied of Mathematics, Chaohu University, Hefei 466001, P. R. China \\ cuiyf@zknu.edu.cn
}

\begin{abstract}
Virtual axis machine tool is widely used in the machining process of complicated curved surface, pointing on the uncertain factors that exists in the virtual axis machine tool system will affect the process accuracy of the virtual axis machine tool, also take the problem that the upper bound of the interference of the actual system are unable to be measured into considering, in this paper, a sliding model control scheme with upper bound adaptive learning based on RBF networks, and the proposed scheme is realized in the MATLAB platform. The simulation results revealed that compared with the traditional sliding model control, the proposed control algorithm has the good performance on position tracking, the error upper bound prediction, chattering reducing, fasting convergence and so forth.
\end{abstract}

Keywords: Virtual axis machine tool, Uncertain factors, RBF, The error upper bound prediction, Chattering

\section{Introduction}

Currently, Virtual axis machine tool is widely used in the machining process of complicated curved surface[1,2],comparing with traditional machine tool, the virtual axis machine tool have the features with big stiffness, high precision, tight structure, small motion inertia, good cutting stability and so forth[3],but the performance of the virtual axis machine tool will be affected by the factors, such as manufacturing and assembly errors, clearances, errors caused by steady state cutting load and the gravity, thermal deformation error and so on[4].

So ,many control schemes have been proposed to solve those, the main method include: error compensation control method[5],model-based adaptive control method and so on[6],the error compensation control method is mainly for a specific virtual axis machine tool, and for a particular error factors such as friction, vibration, gravity, therefore, the error compensation control method does not have versatility, the modelbased adaptive control method is not only difficult to achieve, and also due to its control precision depends on the accuracy of the model, we need to establish accurate mathematical model of virtual axis machine tool in advance. Because of the complexity of the virtual axis machine tool and uncertainty of interference in machining, establish its mathematical model is very difficult, and the model is often very difficult to be used in the actual.

Because the virtual axis machine tool is a highly nonlinear system, there are a lot of interference factors, so conventional control strategy is difficult to meet the requirements of the control, sliding model, sliding mode variable structure can effectively solve the 
nonlinear problem, have strong robustness for the parameter uncertainties and external disturbance[7], thereby it provides a good solution for complex system.

Adaptive sliding mode variable structure control is the combination of sliding mode variable structure control and adaptive control, which is a new type of control strategy to solve the problem of parameter uncertain systems control, many scholars have a lot of research work in this aspect, some solutions are obtained[8],in the literature[9] ,the adaptive backstepping and sliding mode variable structure control method is to combine to realize the adaptive sliding mode variable structure control, but this kind of algorithm is just for linear systems.

In the literature[10], a dynamic adaptive variable structure controller is designed, realized control of nonlinear systems with uncertainty and unknown disturbance robust, but it is only for a class of linearization of nonlinear minimum phase systems.

In general, the sliding mode control system must know the upper bound of the uncertainty ,but the interference value upper bound of the actual system generally cannot be measured[11].So, in this paper, a sliding model control scheme with upper bound adaptive learning based on RBF networks has been proposed, in the scheme ,RBF neural network was adopted to realize adaptive learning on the upper bound of the interference, and the chattering phenomenon existing in the sliding mode control can effectively be reduced.

\section{Control System for Virtual Axis Machine Tool}

The tool of the virtual axis machine tool has been fixed in a moving platform with 6-DOF, the moving of the tool has been controlled by the moving platform with 6 DOF.

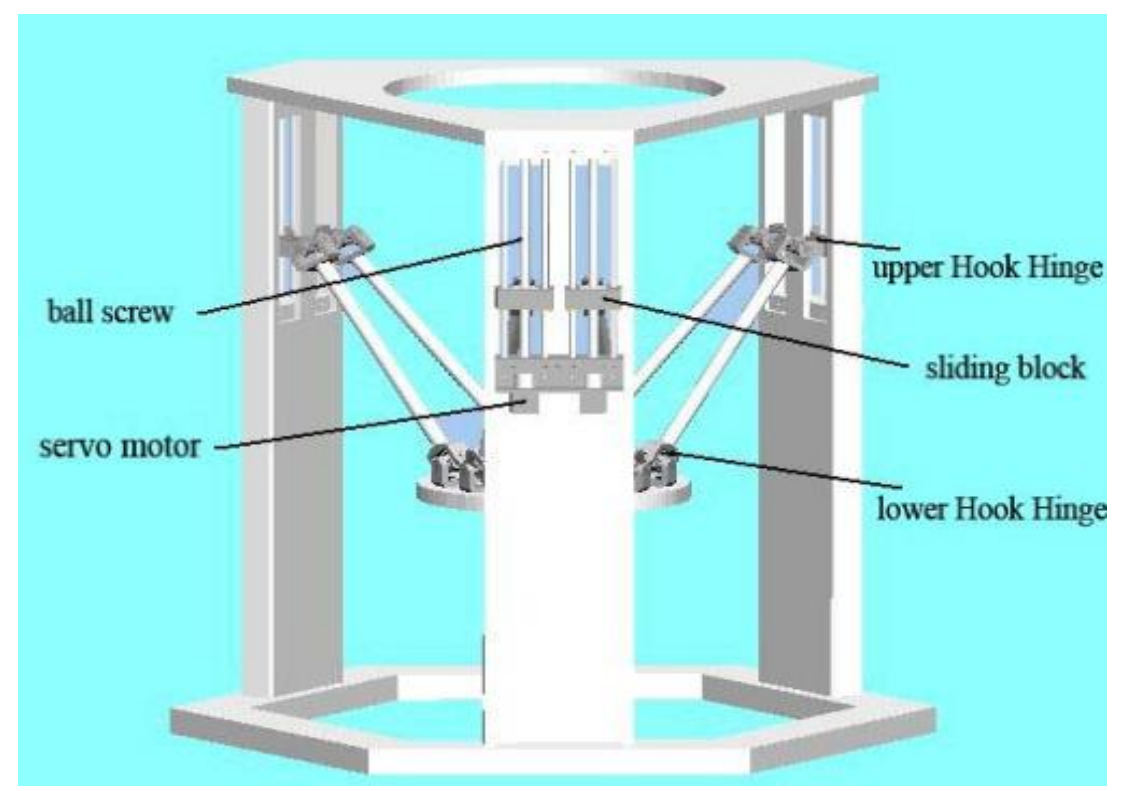

\section{Figure 1. The Parallel Mechanism of a 6-DOF Virtual Axis Machine Tool}

In the 6-DOF virtual axis machine tool, the spatial position of its moving platform is a complex nonlinear function of each joint and there needs decoupling between each branch. So,in this paper we take the sliding model control as the control scheme for virtual axis machine tool, The control system of the virtual axis machine tool by using the sliding model control was shown in Figure 2,in Figure 2, the $\theta_{1 r}, \theta_{2 r}, \ldots 、 \theta_{6 r}$ stand for the desired position of the motor of each joint respectively; $\theta_{1}, \theta_{2}, \ldots, \theta_{6}$ stand for the actual position of the motor of each joint 
respectively, due to its designing process natural decoupled by using sliding mode control system, therefore, each branch adopted the closed-loop control structure respectively, the control system is not sensitive to the parameter variations and external disturbance that existed in the system.

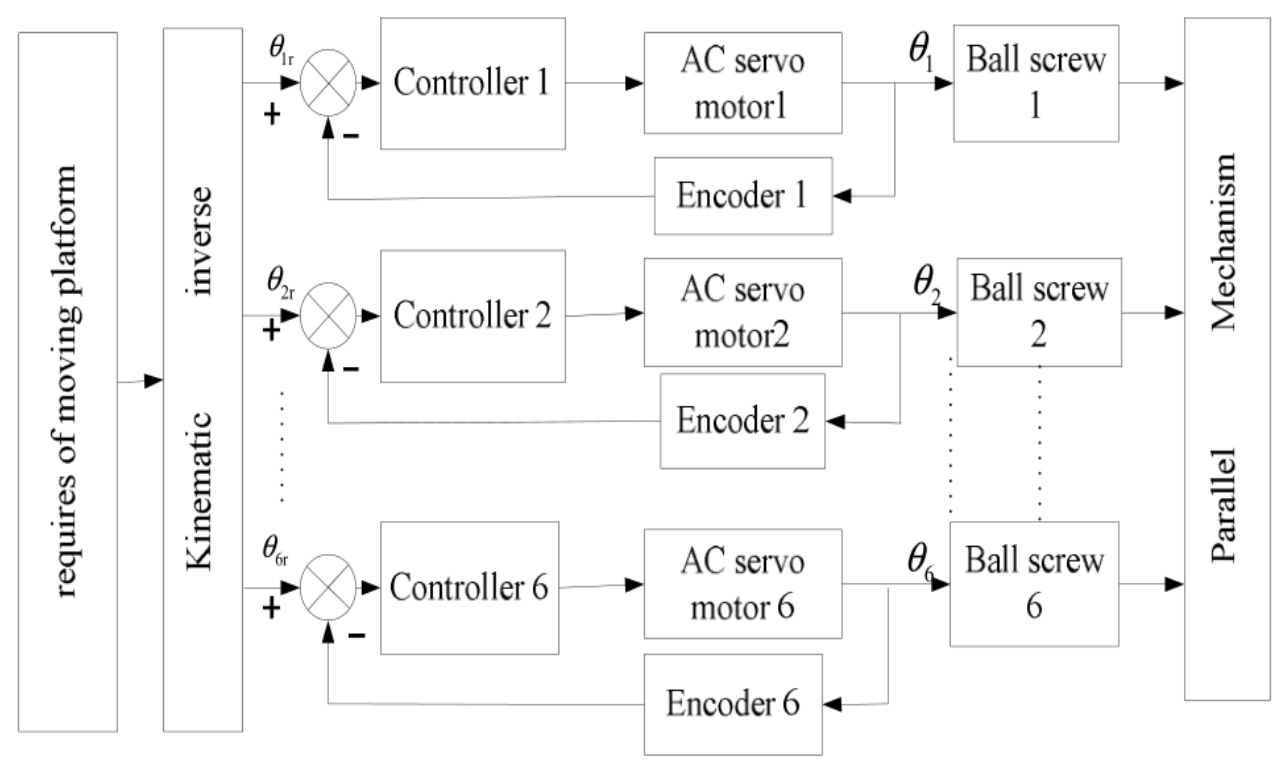

Figure 4. The Control System of the Virtual Axis Machine Tool

\section{System Description}

The main title (on the first page) should begin $13 / 16$ inches (7 picas) from the top edge of the page, centered, and in Times New Roman 14-point, boldface type. Capitalize the first letter of nouns, pronouns, verbs, adjectives, and adverbs; do not capitalize articles, coordinate conjunctions, or prepositions (unless the title begins with such a word). Please initially capitalize only the first word in other titles, including section titles and first, second, and third-order headings (for example, "Titles and headings" — as in these guidelines). Leave two blank lines after the title.

Supposed the control plant as:

$$
\ddot{\theta}=f(\theta, \stackrel{\square}{\theta})+b u+f_{d}
$$

Where $b>0, \theta$ is Angular displacement, $\theta$ is angular velocity, $f(\square)$ and $\mathrm{b}$ is uncertainty, $f_{d}$ is external disturbance, the control input is $u$.

The Equation(1)was rewrite as

$$
b^{-1} \theta-b^{-1} f(\theta, \theta)=u(t)+b^{-1} f_{d}
$$

Supposed $M=b^{-1}, h(\theta, \theta)=-b^{-1} f(\theta, \theta), d(t)=b^{-1} f_{d}$, then Equation(2)has been changed as:

$$
M \theta+h(\theta, \theta)=u(t)+d(t)
$$

Assume that the $M$ and $h(\theta, \dot{\theta})$ are consisted with certainty and uncertainty, then

$$
M=M_{n}+\Delta M
$$




$$
h(\theta, \stackrel{\square}{\theta})=h_{n}(\theta, \stackrel{\square}{\theta})+\Delta h(\theta, \stackrel{\square}{\theta})
$$

Where $M_{n}$ and $h_{n}(\theta, \stackrel{\square}{\theta})$ are certainty, $\Delta M$ and $\Delta h(\theta, \stackrel{\square}{\theta})$ stand uncertainty,

By equation (3)to(5) we can get:

Where

$$
M_{n} \stackrel{\square}{\theta}+h_{n}(\theta, \stackrel{\square}{\theta})=u(t)+\rho(t)
$$

Supposed the nominal model a

$$
\rho(t)=-\Delta M \stackrel{\square}{\theta}-\Delta h(\theta, \stackrel{\square}{\theta})+d(t)
$$

$$
M_{n} \theta+h_{n}(\theta, \stackrel{\square}{\theta})=u_{1}
$$

The total control rule is:

$$
u=u_{1}+u_{0}
$$

By equation(6),

$$
M_{n} \theta+h_{n}(\theta, \theta)=u_{1}+u_{0}+\rho(t)
$$

Where $u_{1}$ stand for the control rule by using nominal model, $u_{0}$ is the control rule of compensation,

Assume the upper bound of the uncertainty as $\rho(t)$, then

$$
|\rho(t)|<\bar{\rho}(t)
$$

\section{Design for Controller}

The system has been divided into two part(nominal model and uncertainty),the nominal model part was controlled by state feedback method,the RBF was used to compensate for the uncertainty, the accurate mathematical model is not necessary in the proposed control scheme, it can realize the robust control for the plant with uncertain factors such as external disturbance and parameter changes.

\subsection{Control Rule for the Nominal Model Part}

Supposed:

$$
\left\{\begin{array}{l}
e_{1}=\theta-\theta_{d} \\
e_{2}=\theta-\theta_{d}
\end{array}\right.
$$

where $\theta_{d}$ and $\theta_{d}$ is the desired value of $\theta$ and $\theta$,

Then

$$
\left\{\begin{array}{c}
e_{1}=e_{2} \\
e_{2}=M_{n}^{-1} u_{1}-M_{n}^{-1} h(\theta, \theta)-\theta_{d}
\end{array}\right.
$$

Take

$$
\begin{gathered}
v=M_{n}^{-1}\left[u_{1}-h_{n}(\theta, \theta)\right]-\theta_{d} \\
{\left[\begin{array}{l}
\square \\
e_{1} \\
\square \\
e_{2}
\end{array}\right]=\left[\begin{array}{ll}
0 & 1 \\
0 & 0
\end{array}\right]\left[\begin{array}{l}
e_{1} \\
e_{2}
\end{array}\right]+\left[\begin{array}{l}
0 \\
1
\end{array}\right] v}
\end{gathered}
$$


Then

$$
e=A e+B v
$$

Where $A=\left[\begin{array}{ll}0 & 1 \\ 0 & 0\end{array}\right], B=\left[\begin{array}{l}0 \\ 1\end{array}\right]$.

Supposed

Where

$$
\left[\begin{array}{ll}
-k_{1} & -k_{2}
\end{array}\right]\left[\begin{array}{l}
e_{1} \\
e_{2}
\end{array}\right]=-k_{1} e_{1}-k_{2} e_{2}=K e=v
$$

$$
K=\left[\begin{array}{ll}
-k_{1} & -k_{2}
\end{array}\right] \quad e=\left[\begin{array}{l}
e_{1} \\
e_{2}
\end{array}\right] .
$$

The control rule is

$$
u_{1}=M_{n}\left(K e+\stackrel{\mathbb{\theta}}{\theta_{d}}\right)+h_{n}(\theta, \stackrel{\square}{\theta})
$$

Put equation (17)into (15),we can get

$$
\left[\begin{array}{c}
e_{1} \\
e_{2}
\end{array}\right]=\left[\begin{array}{ll}
0 & 1 \\
0 & 0
\end{array}\right]\left[\begin{array}{l}
e_{1} \\
e_{2}
\end{array}\right]+\left[\begin{array}{l}
0 \\
1
\end{array}\right]\left[\begin{array}{ll}
-k_{1} & -k_{2}
\end{array}\right]\left[\begin{array}{l}
e_{1} \\
e_{2}
\end{array}\right]
$$

Then

$$
e=A_{1} e
$$

Where $A_{1}=A+B K$.

By designing state feedback gain the closed loop poles can be the arbitrary point in the state space by equation(20).

\subsection{The design of the Sliding Mode Compensator When Upper Bound is Known}

When there existed uncertainty,by equation(10),

$$
\left\{\begin{array}{c}
e_{1}=e_{2} \\
e_{2}=M_{n}^{-1}\left[u_{1}-h_{n}(\theta, \theta)\right]-\theta_{d}+M_{n}^{-1}\left[u_{0}+\rho(t)\right]
\end{array}\right.
$$

Put equation(14)and equation(17)into equation(21), then

$$
\left\{\begin{array}{c}
e_{1}=e_{2} \\
e_{2}=K e+M_{n}^{-1}\left[u_{0}+\rho(t)\right]
\end{array}\right.
$$

Define the sliding model function as:

$$
s=c e_{1}+e_{2}
$$

then

$$
s=c e_{1}+e_{2}=c e_{2}+e_{2}
$$

Define the Lyapunov function as follows: 


$$
V=\frac{1}{2} s^{2}
$$

The

$$
u_{0}=\left\{\begin{array}{cc}
\frac{1}{s M_{n}^{-1}} \omega & |s| \neq 0 \\
0 & |s|=0
\end{array}\right.
$$

Where

$$
\begin{gathered}
\omega=-s c e_{2}-s K e-\left|s M_{n}^{-1}\right| \bar{\rho}(t) \\
\left.V=s s=s\left[c e_{2}+K e+M_{n}^{-1}\left(u_{0}\right)+\rho(t)\right)\right] \\
=s\left(c e_{2}+K e\right)+\left(s M_{n}^{-1}\right) u_{0}+s M_{n}^{-1} \rho(t) \\
=s\left(c e_{2}+K e\right)+\omega+s M_{n}^{-1} \rho(t) \\
=-\left|s M_{n}^{-1}\right| \bar{\rho}(t)+s M_{n}^{-1} \rho(t) \leq-\left|s M_{n}^{-1}\right| \bar{\rho}(t)+\left|s M_{n}^{-1} \| \rho(t)\right| \\
=\left|s M_{n}^{-1}\right|(|\rho(t)|-\bar{\rho}(t))<0
\end{gathered}
$$

The equation(26)changed as:

$$
u_{0}=\left\{\begin{array}{cl}
-\left|M_{n}\right|\left(c e_{2}+K e-\operatorname{sgn}(s) \operatorname{sgn}\left(M_{n}\right) \rho(t)\right) & |s| \neq 0 \\
0 & |s|=0
\end{array}\right.
$$

\section{The Sliding Mode Compensator based on RBF Upper Bound Adaptive Learning}

The input of the RBF networks is $x=[\theta, \dot{\theta}]$, Output is the estimation of the upper bound of uncertain parameters $\hat{\dot{\rho}}(x, \omega)$,

$$
\hat{\dot{\rho}}(x, \omega)=\hat{\omega}^{\mathrm{T}} \phi(x)
$$

Where $\hat{\omega}^{\mathrm{T}}$ is weight value of the $\mathrm{RBF}, \phi(x)$ is the gaussian function[12],

$$
\phi_{i}(x)=\exp \left(-\frac{\left\|x-m_{i}\right\|^{2}}{\sigma_{i}^{2}}\right) \quad i=1,2,3
$$

Where $m_{i}$ is central position of the ith nerve cell, $\sigma_{i}$ is the width of the ith neurons,

The equation(29)changed as

$$
u_{0}=\left\{\begin{array}{cc}
-\left|M_{n}\right|\left(c e_{2}+K e\right)-\operatorname{sgn}(s) \operatorname{sgn}\left(M_{n}\right) \hat{\bar{\rho}}(t) & |s| \neq 0 \\
0 & |s|=0
\end{array}\right.
$$

Assumption 1 the optimal weights $\omega^{*}$ of RBF meet:

$$
\omega^{* T} \phi(x)-\bar{\rho}(t)=\varepsilon(x)<\varepsilon_{1}
$$

Assumption 2 the upper bound of the uncertain parameters meet:

Define the Lyapunov function as

$$
\bar{\rho}(t)-|\rho(t)|>\varepsilon_{0}>\varepsilon_{1}
$$




$$
V=\frac{1}{2} s^{2}+\frac{1}{2} \eta^{-1} \tilde{\omega}^{T} \tilde{\omega}
$$

where $\tilde{\omega}=\omega^{*}-\hat{\omega}$

Adjust the weights online by using the adaptive algorithm, take

$$
\begin{aligned}
& \dot{\hat{\omega}}=\eta\left|s M_{n}^{-1}\right| \phi(x) \\
& \eta=\left|M_{n}^{-1}\right|\left(\varepsilon_{0}-\varepsilon_{1}\right)>0
\end{aligned}
$$

Then

$$
\begin{aligned}
& \dot{V}=s \dot{s}-\eta^{-1} \tilde{\omega}^{T} \dot{\hat{\omega}} \\
& =s\left(c e_{2}+K e\right)+\omega+s M_{n}^{-1} \rho(t)-\eta^{-1} \tilde{\omega}^{T} \dot{\hat{\omega}} \\
& =-\left|s M_{n}^{-1}\right| \hat{\omega}^{T} \phi(x)+s M_{n}^{-1} \rho(t)-\eta^{-1} \tilde{\omega}^{T} \dot{\hat{\omega}} \\
& =-\left|s M_{n}^{-1}\right|\left(\hat{\omega}^{T} \phi(x)+\bar{\rho}(t)-\bar{\rho}(t)\right)+s M_{n}^{-1} \rho(t)-\eta^{-1} \tilde{\omega}^{T} \dot{\hat{\omega}} \\
& \leq-\left|s M_{n}^{-1}\right|\left(\hat{\omega}^{T} \phi(x)+\bar{\rho}(t)\right)-\left|s M_{n}^{-1}\right|(\bar{\rho}(t)-|\rho(t)|)-\eta^{-1} \tilde{\omega}^{T} \dot{\hat{\omega}} \\
& =-\left|s M_{n}^{-1}\right|\left(\hat{\omega}^{T} \phi(x)-\hat{\omega}^{* T} \phi(x)+\varepsilon(x)\right)-\left|s M_{n}^{-1}\right|(\bar{\rho}(t)-|\rho(t)|) \\
& \quad \quad-\left(\hat{\omega}^{T}-\hat{\omega}^{* T}\right)\left|s M_{n}^{-1}\right| \phi(x) \\
& \quad=-\left|s M_{n}^{-1}\right| \varepsilon(x)-\left|s M_{n}^{-1}\right|(\bar{\rho}(t)-|\rho(t)|) \\
& \quad \leq\left|s M_{n}^{-1} \| \varepsilon(x)\right|-\left|s M_{n}^{-1}\right|(\bar{\rho}(t)-|\rho(t)|) \\
& =\left|s M_{n}^{-1}\right|(|\varepsilon(x)|-(\bar{\rho}(t)-|\rho(t)|))
\end{aligned}
$$

By Assumption 1

$$
|\varepsilon(x)|<\varepsilon_{1}
$$

By Assumption 2

$$
\mid-(\bar{\rho}(t)-|\rho(t)|)<-\varepsilon_{0}
$$

Then

$$
|\varepsilon(x)|-(\bar{\rho}(t)-|\rho(t)|)<\varepsilon_{1}-\varepsilon_{0}
$$

then

$$
\dot{V} \leq-\left|s M_{n}^{-1}\right|\left(\varepsilon_{0}-\varepsilon_{1}\right)=-\eta|S| \leq 0
$$

\section{Numerical Simulation}

We has implemented the proposed algorithm in the branch Virtual Axis Machine Tool in the Matlab simulation platform to verify the proposed algorithm's validity[13], supposed the control plant as:

$$
\left\{\begin{array}{c}
\dot{x}_{1}=x_{2} \\
\dot{x}_{2}=-25 x_{2}+133 u+d(t)
\end{array}\right.
$$

The input of the RBF is $\left[\begin{array}{ll}x_{1} & x_{2}\end{array}\right]$, take the initial value of $w$ as $\left[\begin{array}{lll}0.1 & 0.1 & 0.1\end{array}\right]^{T}$, the initial value of $\mathrm{m}$ are random between $-1 \sim 1$, take the initial value of $\sigma$ as $\left[\begin{array}{lll}0.2 & 0.2 & 0.2\end{array}\right]^{T}$, the initial state of the system is $\left[\begin{array}{ll}0.5 & 0\end{array}\right], d(t)$ is $0.5 \sin (2 \pi t)$, the simulations are shown in the Figure 3 to Figure 8,the Figure 3 is position tracking by using sliding model control without compensation, the Figure 4 is position tracking of sliding model control with upper bound adaptive learn compensation, the Figure 5 is 
position tracking error by using sliding model control without compensation, the Figure 6 is position tracking error of sliding model control with upper bound adaptive learn compensation, from Figure 3 to 6 we can get that the proposed control scheme based on upper bound adaptive learn compensation have the features of good position tracking and fasting convergence, the Figure 7 is the control input of the sliding model control without compensation,the Figure 8 is the control input of the sliding model control with upper bound adaptive learn compensation, from Figure 7 to 8 we can get that the changing of control input is more smooth than the sliding model control without compensation, then the chattering are reduced.

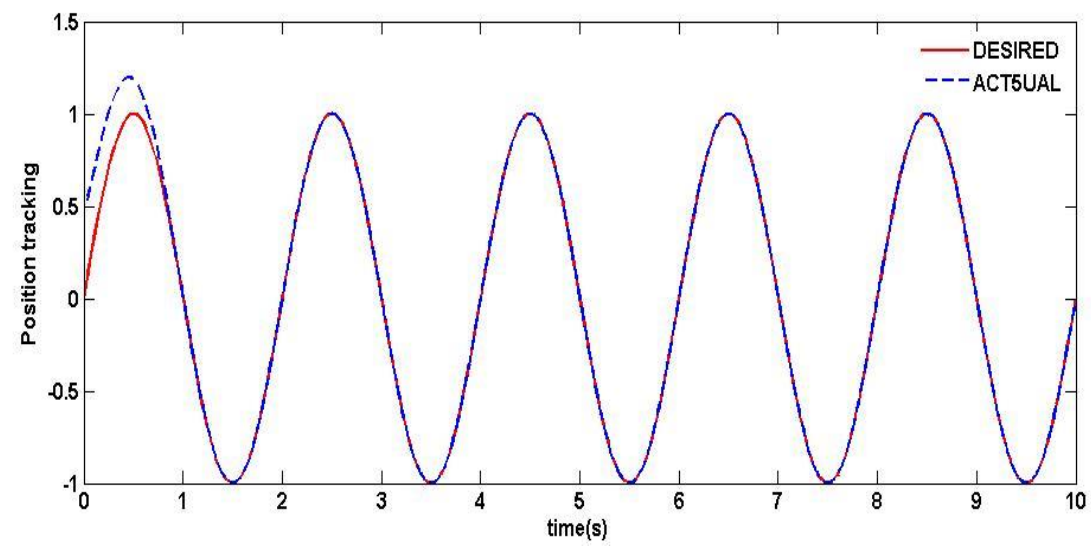

Figure 3. Sliding Model Control Position Tracking Without Compensation

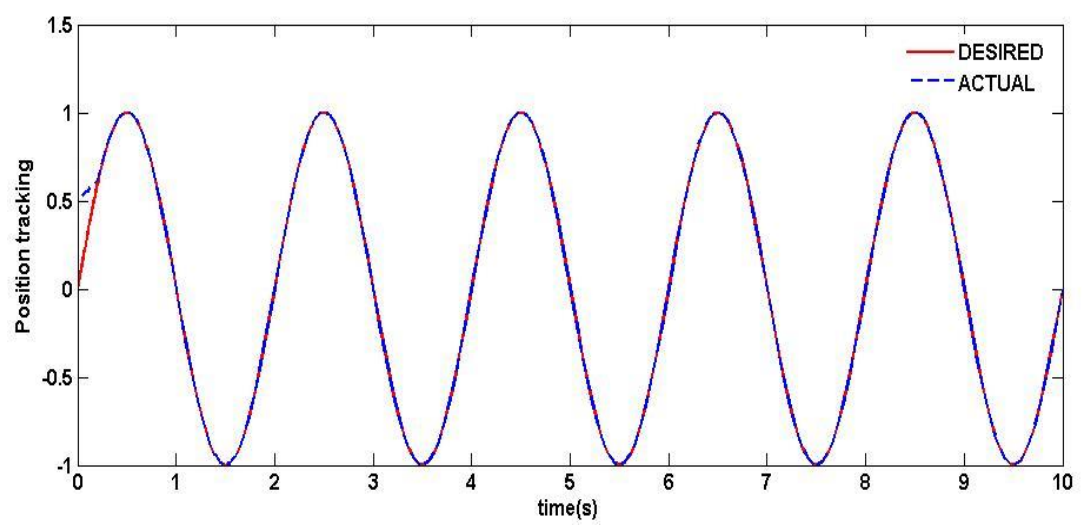

Figure 4. Sliding Model Control Position Tracking with Upper Bound Adaptive Learn Compensation 


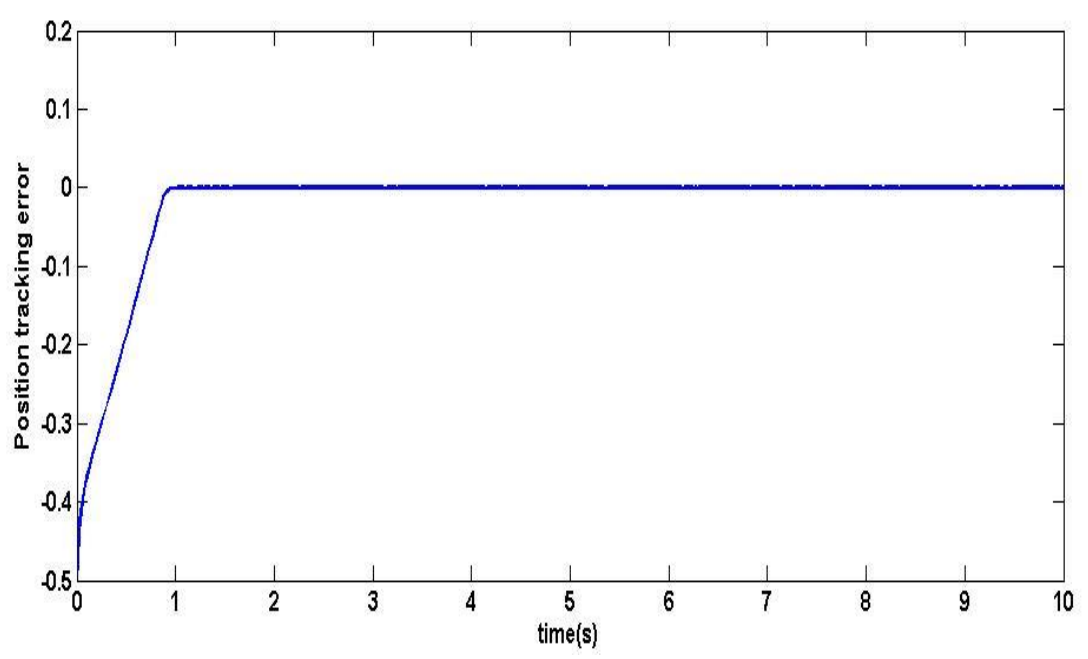

Figure 5. Sliding Model Control Position Tracking Error without Compensation

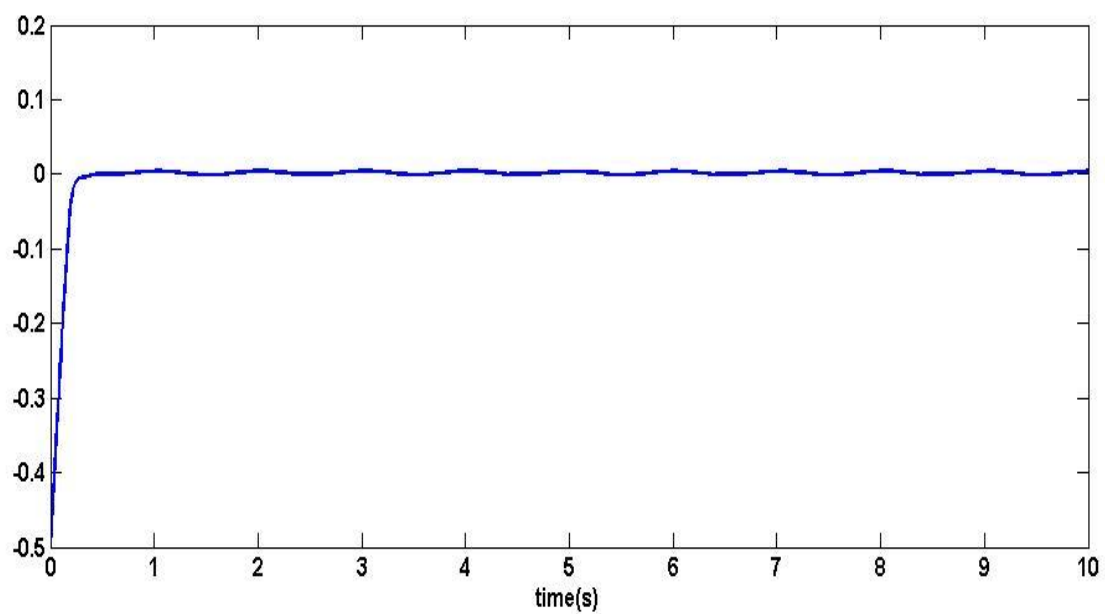

Figure 6. Sliding Model Control Position Tracking Error with Upper Bound Adaptive Learn Compensation

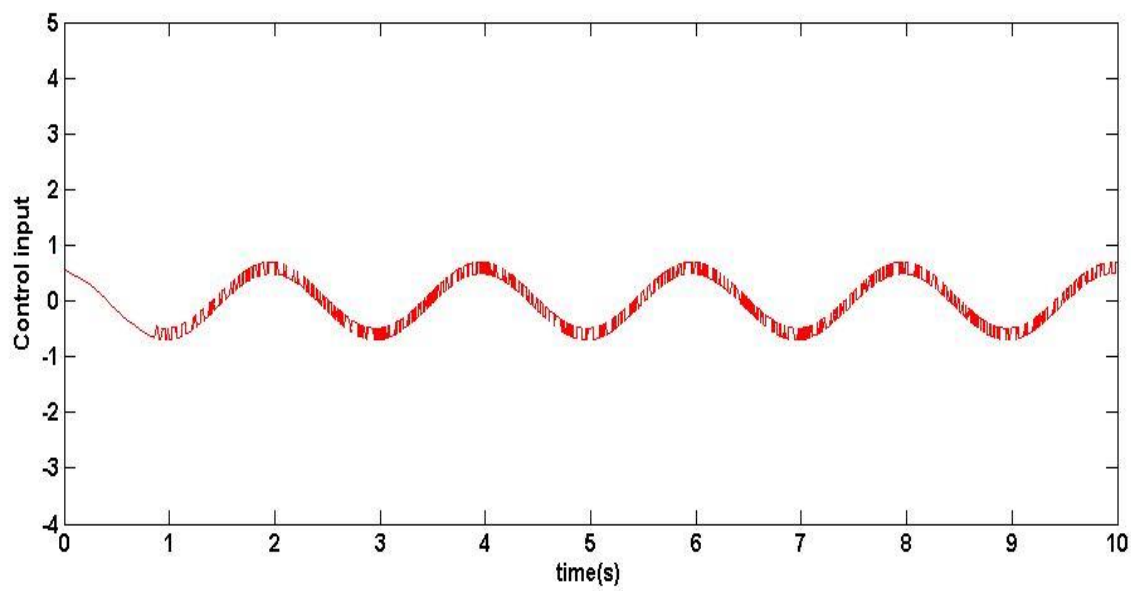

Figure 7. Control Input of the Sliding Model Control without Compensation 


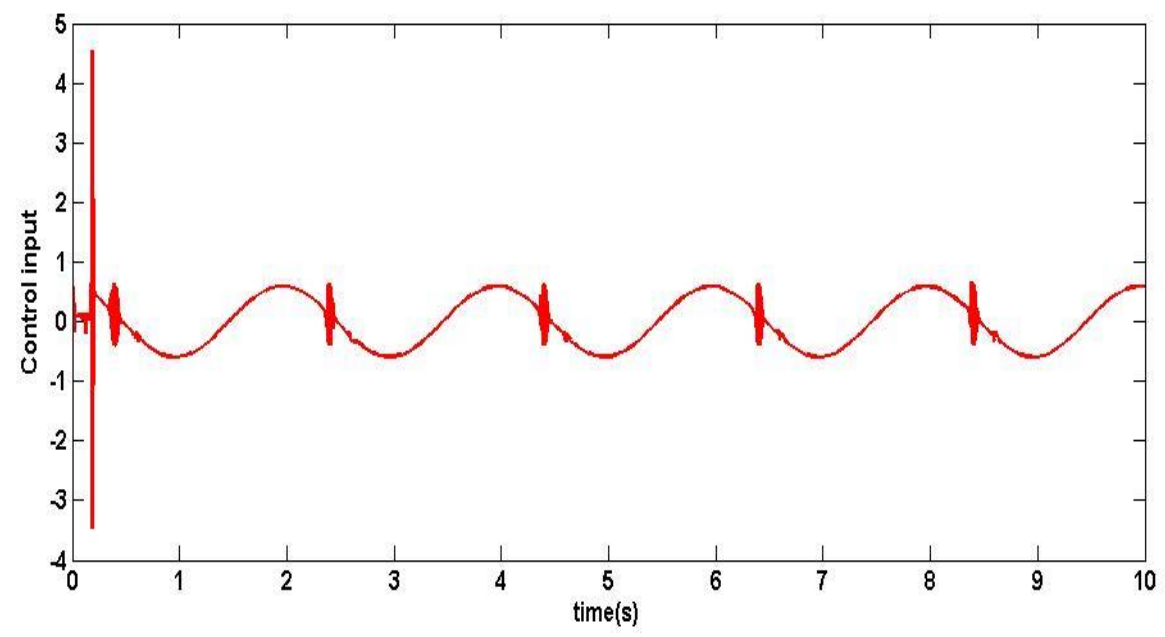

\section{Figure 8. The Control Input of the Sliding Model Control with Upper Bound Adaptive Learn Compensation}

\section{Conclusions}

Virtual axis machine tool is widely used in the machining process of complicated curved surface, it has the features with big stiffness, high precision, tight structure, small motion inertia, good cutting stability and so forth, but the performance of the virtual axis machine tool will be affected by the uncertainty , take the problem that the upper bound of the interference of the actual system are unable to be measured into considering, in this paper, a sliding model control scheme with upper bound adaptive learning based on RBF networks, and the proposed scheme is realized in the MATLAB platform. The simulation results revealed that compared with the traditional sliding model control, the proposed control algorithm has the following good performance.

(1)The proposed control scheme based on upper bound adaptive learn compensation have the features of good position tracking and fasting convergence.

(2)The changing of control input is smoother than the sliding model control without compensation, then the chattering are reduced.

\section{Acknowledgements}

This work is supported by the Natural Science Foundation of Chaohu University(No. XLY-201503), the University Natural Science Foundation of Anhui(No. KJ2015A270).

\section{References}

[1] X. Liu, J. Wang and L. Wang, "Optimal Kinematic Design of the 2-DoF Translational Parallel Mechanism in a 5-Axis Gantry Machine Tool", Proceedings of the 5th Chemnitz Parallel Kinematics Seminar, Chemnitz, Germany, (2006).

[2] X.-J. Liu, X. Tang and J. Wang, "A Novel 2-DOF Parallel Mechanism based Design of a new 5-axis Hybrid Machine Tool", Proceedings of IEEE International Conference on Robotics and Automation (ICRA'2003), Taipei, Taiwan, (2003).

[3] G. Gao, Q. Ding and W. Wang, "The application of RBF neural network sliding mode control on parallel robot", Industrial Instrumentation \& Automation, no. 2, (2012), pp. 35-39.

[4] M. Weck and D. Staimer, "Accuracy issues of Parallel Kinematic Machine Tools", Proceedings of the Institution of Mechanical Engineers, Part K: Journal of Multy-Body Dynamics, vol. 216, pp. 51-57, (2002).

[5] S. Ibaraki, T. Okuda and Y. Kakino, "Compensation of Gravity-Induced Errors on a Hexapod-Type Parallel Kinematic Machine Tool”, JSME International Journal, Series C: Mechanical Systems, Machine Elements and Manufacturing, vol. 47, (2004), pp. 160-167 
[6] C.-G. Li, H.-S. Ding and P.-D. Wu, "Application of Adaptive Control in Parallel Machine Tool", Machine Tool \& Hydraulics, no. 11, (2004), pp. 25-27.

[7] G. Gao, W. Wang and Q. Ding, "Synchronization Sliding Mode Control for Agricultural Parallel Robot", Transactions of the Chinese Society of Agricultural Machinery, vol. 43, no. 9, (2012), pp. 173-178,196.

[8] G.-Q. Gao, W.-J. Xia and Q. Song, "Adaptive sliding control for integral switching surface and its application in parallel robot”, Machinery Design \& Manufacture, no. 2, (2012), pp. 146-148.

[9] R.H. Sira and S.O. Llanes, "Adaptive dynamical sliding mode control via backstepping”, Proceedings of the 32nd IEEE Conference on Decision and Control, SanAntonio, United States, (1993).

[10] M.R. Bolivar, A.S.I. Zinober and H. Sira-Ramirez, "Dynamic Adaptive sliding mode output tracking control of a class of nonlinear systems", International Journal of Robust and Nonlinear Control, vol. 7 , no. 4, (1995), pp. 387-405.

[11] S. Wang, H. Wang and L. Zhao, "Optimal Neural Network-based Sliding Mode Control for Robot Manipulators with Uncertainties, Machine Tool \& Hydraulics, vol. 37, no. 7, (2009).

[12] K. Dai, G. Wang and C. Zhao, "A prediction model for energy consumption of building based on PCA and RBF", Journal of Fuzhou University(Natural Science Edition), vol. 43, no. 4, (2015, pp. 512-516).

[13] J. Cao, C. Tong, D. Hongtao and H. Li, "Simulation of Induction Motor of Sliding Mode Based on Matlab/Simulink", Journal of North China University of Technology, vol. 27, no. 1, (2015).
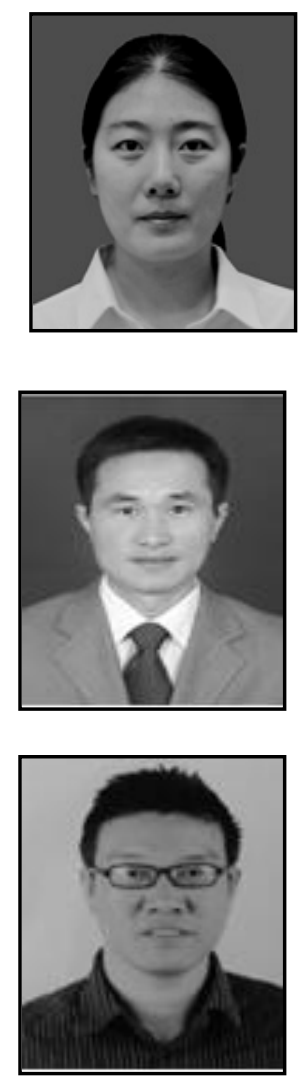

\section{Authors}

Yu Zhao, she received the Master degree in department of mathematics from Southeast University, in 2011. Currently, she is a lecturer at School of Basic Education, Jiangsu Food \& Pharmaceutical Science College. Her research interests include different aspects of Applied of Mathematics and Control Engineering..

Yongfeng Cui, he received the BS degree in Computer Science and Technology from Henan Normal University and the MS degree in Computer Application Technology from Huazhong University of Science and Technology, China in 2000 and 2007 respectively. He is currently researching on Computer Application Technology (CAT).

Miaochao Chen, he received the Master degree in department of mathematics from Southeast University, in 2011. Currently, he is a lecturer at School of Applied of Mathematics, ChaoHu University. His research interests include different aspects of Partial Differential Equation and Control Engineering. 
International Journal of Control and Automation

Vol. 9, No. 9 (2016) 